[Article]

\title{
甘氨酸二肽分子酰胺-I带光谱与结构相关性
}

\author{
蔡开聪 ${ }^{1,2, *}$ 郑 轩 ${ }^{1,2}$ 刘亚男 ${ }^{1,2}$ 留珊红 ${ }^{1,2}$ 杜芬芬 ${ }^{1,2}$ \\ ('福建师范大学化学与化工学院, 福州 350007; '2福建省理论与计算化学重点实验室, 福建 厦门 361005)
}

\begin{abstract}
摘要: 系统探索了蛋白质二肽模型分子一一甘氨酸二肽 (GLYD)在气相与水溶液中的结构与光谱特性。从分 子动力学轨迹中提取具有代表性结构的 GLYD- $\mathrm{D}_{2} \mathrm{O}$ 聚集体的瞬态结构开展简正模式分析, 获取了对蛋白质二 级结构敏感的酰胺-I带的振动光谱参数, 建立起振动光谱与特征基团结构间的相关性。将溶剂作用以静电势 场的形式投影至二肽分子骨架中, 与酰胺-1带在气/液相中的频率差相关联, 并引入酰胺- 1 带简正模式随二级 结构变化的规律, 将各个构象态可能存在的振动耦合包含在内, 构建具有二级结构敏感性的静电频率转换 图, 实现溶液相中多肽骨架酰胺-I带的快速准确预测。
\end{abstract}

关键词: 甘氨酸二肽; 酰胺-I带; 振动光谱; 静电频率图

中图分类号: 0641

\section{Correlation between Amide-I Spectra and Structural Features of Glycine Dipeptide}

\author{
CAI Kai-Cong ${ }^{1,2, *}$ ZHENG Xuan ${ }^{1,2}$ LIU Ya-Nan ${ }^{1,2} \quad$ LIU Shan-Hong ${ }^{1,2} \quad$ DU Fen-Fen ${ }^{1,2}$ \\ $\left({ }^{1}\right.$ College of Chemistry and Chemical Engineering, Fujian Normal University, Fuzhou 350007, P. R. China; \\ ${ }^{2}$ Fujian Provincial Key Laboratory of Theoretical and Computational Chemistry, Xiamen 361005, Fujian Province, P. R. China)
}

\begin{abstract}
Structural and spectroscopic features of a model dipeptide, glycine dipeptide (GLYD), were systematically investigated in the gas phase and in aqueous solution. Normal mode analysis was performed on the representative GLYD- $\mathrm{D}_{2} \mathrm{O}$ clusters selected from molecular dynamics (MD) trajectory for the vibrational parameters of amide-I mode, which is known to be sensitive to the secondary structure of proteins. On this basis, the correlation between the vibrational spectrum and the structural features of specific groups in the polypeptide was constructed. The electrostatic potential from the solvent molecules was calculated and projected onto the backbone of GLYD, and related to the amide-I frequency difference for GLYD in gas phase and solution phase. The secondary structure-dependent normal mode amide-I frequency database was also introduced for the consideration of the possible vibrational coupling that is intrinsically included in GLYD conformers. An electrostatic frequency map with secondary structural sensitivity was then built for the fast and accurate vibrational frequency prediction of the amide-I vibrational band for polypeptides in solution.
\end{abstract}

Key Words: Glycine dipeptide; Amide-I band; Vibrational spectrum; Electrostatic frequency map

1 引言

要发挥正常的物理、化学以及生物学功能, 蛋白质需要通过大量氢键、范德华力和疏水作用
等非共价相互作用来正确折叠形成一个特定构 型。组织中特定蛋白质如果发生错误折叠, 将会 引起空间构象变化, 进而发生自组装形成难溶的

Received: December 24, 2015; Revised: February 29, 2016; Published on Web: February 29, 2016.

*Corresponding author. Email: ckc1117@fjnu.edu.cn; Tel: +86-591-22868161.

The project was supported by the National Natural Science Foundation of China (21103021) and Education Department of Fujian Province of China (JA13063).

国家自然科学基金(21103021)和福建省高校杰出青年科学人才培育计划(JA13063)资助项目

(c) Editorial office of Acta Physico-Chimica Sinica 
纤维聚集体, 引发如阿尔茨海默病、II 型糖尿病、 汉庭顿舞蹈症等所谓的 “蛋白质构象病” 1, 从而 给公共卫生带来巨大压力, 引发重大社会问题 ${ }^{2}$ 。 要从分子水平上研究蛋白质发挥作用的机制, 需 要深化对蛋白质构象态即二级结构变化的结构动 力学信息的认识, 了解其可能的构象态布居倾 向。作为一级结构和三维空间结构之间的重要桥 梁, 蛋白质二级结构的预测对于理解溶液相中蛋 白质空间构型、折叠机制以及蛋白质所产生的功 能具有重要的理论价值, 而且对于揭示蛋白质结 构的改变对生物体的影响乃至预防疾病的产生有 着重要的指导作用 ${ }^{3,4}$ 。

蛋白质及多肽的骨架上酰胺- $\mathrm{I}$ 带(主要为 $\mathrm{C}=\mathrm{O}$ 的伸缩振动)在中红外区域具有强烈的振动吸收, 其特征吸收峰对分子骨架二级结构的变化十分敏 感, 因此广泛用作蛋白质及多肽的结构探针 ${ }^{5}$ 。酰 胺-I 带的特征振动吸收谱带位于 $1600-1700 \mathrm{~cm}^{-1}$, 对于特定的二级结构可以用 “指纹” 识别的方法 进行鉴定：如 $\alpha$ 螺旋在 $1655 \mathrm{~cm}^{-1}$ 处有一个特征吸 收峰, 且随着螺旋链的增长而红移; 而 $\beta$ 折叠则出 现明显的双峰, 其在 $1630 \mathrm{~cm}^{-1}$ 处有个较强吸收 峰, 在 $1685 \mathrm{~cm}^{-1}$ 处吸收峰相对较弱 ${ }^{6-9}$ 。蛋白质骨 架酰胺吸收带的零级频率预测一直是一个难题, 由于酰胺单元的跃迁偶极矩之间存在振动耦合作 用, 其振动光谱表现为一个较宽的吸收带, 使得 传统的一维红外光谱学手段难以有效地将各个组 分区分开。为此, 人们常常引入同位素取代的方 法, 如将酰胺- $\mathrm{I}$ 带中 $\mathrm{C}=\mathrm{O}$ 基团修饰为 ${ }^{13} \mathrm{C}={ }^{18} \mathrm{O}$, 从 而使其频率发生约 $60 \mathrm{~cm}^{-1}$ 的红移, 剥离出酰胺-I 带的光谱区域, 从而实现特定位点光谱和结构相 关性解析 ${ }^{10,11}$ 。

许多重要的生命过程都是在溶液环境下进行 的, 要发展蛋白质结构检测方法, 需要在微观层 面上描述蛋白质体系在溶液相中的动态结构以及 包括溶剂在内的微环境。飞秒激光二维红外光谱 等实验新技术的发展使得人们在更小更短暂的时 空领域能够观测到包含微观世界中蛋白质结构涨 落信息的实验信号 ${ }^{12-18}$, 而这些实验信号需要在理 论层面上发展新方法来深入认识和解析溶液中蛋 白质结构及相应的酰胺振动吸收带的光谱特性。 在水溶液中, 溶质一溶剂间的静电相互作用占据了 主导地位, 因此人们提出了用于实现酰胺-I 带振动 频率快速预测的静电频率转换图 ${ }^{9,19-26}$ 。该模型将溶
剂作用以静电势场的形式投影至多肽骨架中，与 酰胺-I 带在气相和液相中的频率差相关联, 通过分 子所处的静电势场环境, 实现酰胺-I带振动光谱的 快速准确预测。静电频率转换图的构建主要基于 模型分子-氮甲基乙酰胺(NMA) 以及非天然氨基酸 模型分子 (NEPA $)^{27-29}$ 。尽管多肽骨架对酰胺-I 带光 谱频率具有显著的影响, 针对具有二级结构的多 肽乃至寡聚肽所构建的模型仍少有报道, 因为要 考察特定结构多肽的酰胺吸收带之间的振动耦 合, 需要通过较为复杂的跃迁偶极耦合作用才能 有效表征多肽链中酰胺-I带的光谱特性 ${ }^{21,23,26,30}$, 更 为经验和简化的方法有待开发。

本文开展分子动力学模拟, 获取了甘氨酸二 肽(GLYD)在水溶液中的全原子运动轨迹, 探究其 动态结构及存在的溶质一溶剂相互作用。从动力学 瞬态结构中提取第一溶剂化层中具有代表性的溶 质一溶剂聚集体, 开展简正模式分析, 了解酰胺-I 带振动光谱参数及溶剂对其所产生的影响。探索 气相中酰胺-I带频率的二级结构依赖性, 了解各个 构象态下两个酰胺-I带的特征简正振动频率, 将各 个构象态中可能存在的振动耦合考虑在内。进一 步将溶剂作用以静电势场的形式投影至酰胺单元 上, 辅以酰胺-I带简正模式的二级结构依赖性, 从 而构建静电频率转换模型, 实现多肽分子骨架酰 胺-I带光谱的快速准确预测。

\section{2 计算方法}

\section{1 分子动力学模拟}

借助 NAMD 软件 ${ }^{31}$ 开展全原子分子动力学模 拟, 探索 GLYD 在重水中的结构动力学特性。 GLYD 采用 CHARMM 力场 (版本号: c35b2) 描 述 ${ }^{32}$, 水溶液则采用 TIP3P 模型描述 ${ }^{33}$ 。研究体系 为 1 个 GLYD 分子和 2279 个重水分子组成的 $4.2 \mathrm{~nm}$ 的立方体盒子。体系设置了周期性边界环境, 长 程静电作用采用了 particle mesh Ewald (PME) 方法 计算, 非键相互作用的截断距离设定为 $1.2 \mathrm{~nm}$ 。

体系采用共轭梯度法进行了 10000 步的能量最 小化, 排除了可能的高能量和空间重叠构型, 随 后逐步升温至室温 $(298 \mathrm{~K})$ 。在恒温恒压系综(NPT) 下采用 Nosé-Hoover Langevin piston 方法进行了分 子动力学模拟, 在 $298 \mathrm{~K}$ 温度下以 $5 \mathrm{fs}$ 的步长采集 了 $1 \mathrm{~ns}$ 全原子运动轨迹。

\section{2 量子化学计算}


从 $1 \mathrm{~ns}$ 的全原子运动轨迹中等间隔提取了其中 20 个瞬态结构, 对这 20 个结构分别提取含有重水 个数 $n=1-5$ 的 GLYD- $n \mathrm{D}_{2} \mathrm{O}$ 聚集体(合计 100 个)开 展量子化学计算, 并在 GLYD- $5 \mathrm{D}_{2} \mathrm{O}$ 聚集体外添加 连续极化介质模型 $(\mathrm{PCM})$ 补偿体相中水的影响。在 B3LYP/6-31+G $(d)$ 水平上对这 120 个聚集体进行结 构优化和简正模式分析, 同时借助势能分布分析 (PED) 方法 ${ }^{34}$ ，对酰胺-I带进行指认。

在 B3LYP/6-31+G $(d)$ 水平上对气相中 GLYD 的 骨架二面角 $(\Phi: \angle C N C C ; \quad \Psi: \angle \mathrm{NCCN})$ 进行扫 描, 固定其中一个二面角, 对另一个二面角进行 旋转(步长为 $10^{\circ}$ )。对得到的 1369 个构象异构体进 行几何结构优化和简正模式分析, 获取各个构象 异构体的酰胺-I带的振动频率, 考察其与多肽二级 结构之间的相关性。所有的量子化学计算均在 Gaussian 09 软件 ${ }^{35}$ 下进行。

\section{3 静电频率转换图的构建}

将经过量子化学计算优化的 GLYD- $n \mathrm{D}_{2} \mathrm{O}$ 聚集 体(共 120 个)作为建模样本。选取的样本具有结构 代表性, 又具有电子结构准确性, 成键和非键相 互作用已经通过分子动力学模拟和量子化学计算 隐性地在样本结构特征中表现出来。将其中溶剂 原子看作质点，GLYD骨架上甲基、亚甲基看作联 合原子(电荷集中在中心原子上), 计算其在酰胺单 元的原子位点 $(\mathrm{C} 、 \mathrm{O} 、 \mathrm{~N} 、 \mathrm{H})$ 上所产生的静电势 场，同时引入酰胺-I带简正模式随骨架二面角变化 的特征, 将其与 GLYD 中酰胺-I 带的气/液相频移 相关联:

$$
v_{1}=v_{\mathrm{g}}+\sum_{i=1}^{4} f_{i} \varphi_{i}+f_{\omega} \omega_{(\Phi, \Psi)}
$$

其中, $v_{1}$ 为 GLYD- $n \mathrm{D}_{2} \mathrm{O}$ 聚集体中酰胺-I 带的频率值 (均乘以校正因子 0.974 , 即气相实验值 $v_{\mathrm{g}}$ 与对应的 $\mathrm{C}_{5}$ 构象下在 B3LYP/6-31+G $(d)$ 水平上计算频率值之 比的均值 $)^{36}, v_{\mathrm{g}}$ 为 GLYD 处于 $\mathrm{C}_{5}$ 构象时酰胺-I 带的 气相实验频率值 $\left(1693 \mathrm{~cm}^{-1}\right.$, 酰胺 $-\mathrm{I}_{\mathrm{a}}$ 带; $1707 \mathrm{~cm}^{-1}$, 酰胺 $-\mathrm{I}_{b}$ 带 $)^{37}, f$ 为模型参数, $\varphi$ 为酰胺单元上所产生 的静电势, $\omega$ 为处于不同构象态时 GLYD 中酰胺-I 带频率与气相值 $v_{\mathrm{g}}$ 的差值。通过求解超定方程组, 获得静电频率图转换参数(表 1 )。
将模型参数应用于全原子动力学轨迹中, 得 到修正后的酰胺-I带频率轨迹及其分布态密度 (DOS), 并通过如下线型函数计算得到振动吸收光 谱图,

$$
I(v) \sim \int_{-\infty}^{\infty} \mathrm{e}^{-\mathrm{i}(v-\langle v) t} \times\left\langle\exp \left[\mathrm{i} \int_{0}^{t} \mathrm{~d} \tau \delta v(\tau)\right]\right\rangle\left\{\mathrm{e}^{-t / T_{1}}\right\} \mathrm{d} t
$$

其中, $I(v)$ 为光谱强度, $v$ 为随时间变化的酰胺-I带 的瞬时振动频率, $t$ 为时间, $\delta v(t)=v(t)-\langle v\rangle$, $\langle v\rangle$ 为酰胺-I 带频率的平均值, $T_{1}$ 为酰胺 $-\mathrm{I}$ 带第一激 发态的寿命 $(0.5 \mathrm{ps})^{38}$ 。

\section{3 结果与讨论}

\section{1 水溶液中 GLYD 的微观结构}

借助空间分布函数 (SDF) 和径向分布函数 (RDF)，系统考察 GLYD 的酰胺单元与水分子之间 可能存在的氢键相互作用及其强弱关系(图 1)。从 空间分布函数(图 1C)中可以看出, GLYD 两个酰胺 单元上的 $\mathrm{C}=\mathrm{O}$ 在较短的距离范围内均被蓝色的区 域所包围，表明 $\mathrm{C}=\mathrm{O}$ 上的 $\mathrm{O}$ 原子与水的 $\mathrm{D}$ 原子形 成较强的氢键作用; 而 $\mathrm{N}-\mathrm{H}$ 周围则被红色的区域 包围，表明 $\mathrm{N}-\mathrm{H}$ 中的 $\mathrm{H}$ 原子倾向于和水中的 $\mathrm{O}$ 原 子结合形成氢键。

通过径向分布函数(图 1(A, B)) 可以看出, 两个 酰胺单元上的 $\mathrm{C}=\mathrm{O}$ 和 $\mathrm{N}-\mathrm{H}$ 基团分别与重水中的 $\mathrm{D}$ 和 $\mathrm{O}$ 原子形成较强的氢键作用。其中, $\mathrm{C}=\mathrm{O}$ 中 的 $\mathrm{O}$ 原子与重水中的 $\mathrm{D}$ 原子在距离分别为 $0.175 \mathrm{~nm}$ 处均形成较强的峰 $\left(g(r)_{\mathrm{a}}=1.21 ; g(r)_{\mathrm{b}}=1.25\right)$; 而 $\mathrm{N}-\mathrm{H}$ 的 $\mathrm{H}$ 原子与重水中的 $\mathrm{O}$ 原子在距离分别为(a) $0.195 \mathrm{~nm}$ 和 (b) $0.205 \mathrm{~nm}$ 处形成两个峰 $\left(g(r)_{\mathrm{a}}=\right.$ $\left.0.80 ; g(r)_{\mathrm{b}}=0.69\right)$ 。通过对第一水合层内的 $g(r)$ 进 行积分, 可以得到第一溶剂化层内的溶剂配位数 $(N)$,

$$
N=4 \pi \int_{0}^{R_{\min }} \rho g(r) r^{2} \mathrm{~d} r
$$

其中, $\rho$ 为密度, $R_{\min }$ 为 $g(r)$ 第一个最小值出现的距 离, $r$ 为原子间距离。研究结果表明, GLYD的两 个酰胺单元的 $\mathrm{C}=\mathrm{O}$ 基团第一溶剂化层中围绕着两 个 $\mathrm{D}_{2} \mathrm{O}$ 分子, $\mathrm{N}-\mathrm{H}$ 基团周围围绕着一个 $\mathrm{D}_{2} \mathrm{O}$ 分 子。由于酰胺单元的亲水性及氢键作用, GLYD 在

表 1 甘氨酸二肽中酰胺-I 带的静电频率转换图参数

Table 1 Parameters of the electrostatic frequency map for the amide-I band of GLYD

\begin{tabular}{cccccc}
\hline & $f_{\mathrm{d}} /\left(\mathrm{cm}^{-1} \cdot \mathrm{V}^{-1}\right)$ & $f_{\mathrm{o}} /\left(\mathrm{cm}^{-1} \cdot \mathrm{V}^{-1}\right)$ & $f_{\mathrm{N}} /\left(\mathrm{cm}^{-1} \cdot \mathrm{V}^{-1}\right)$ & $f_{\mathrm{H}} /\left(\mathrm{cm}^{-1} \cdot \mathrm{V}^{-1}\right)$ & $f_{\mathrm{\omega}} /\left(\mathrm{cm}^{-1} \cdot \mathrm{V}^{-1}\right)$ \\
\hline GLYD & 12.795 & -44.801 & 29.779 & -9.574 & -0.208 \\
\hline
\end{tabular}



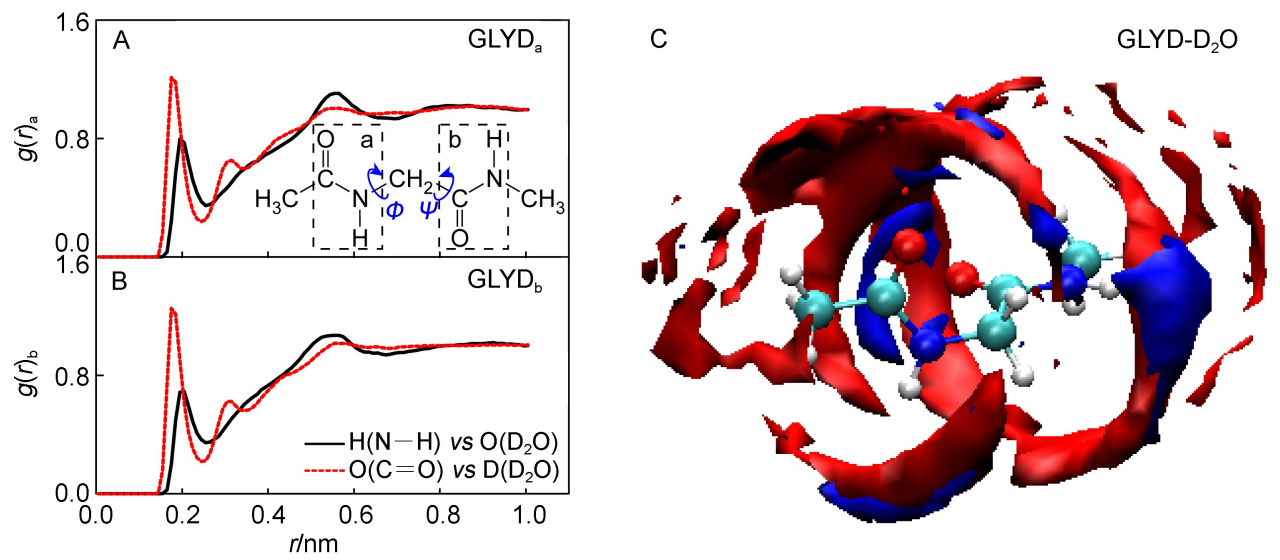

图 1 甘氨酸二肽与水分子形成的径向分布函数 $(A, B)$ 与空间分布函数 $(C)$

Fig.1 Radial distribution function (A, B) and spatial distribution function (C) between GLYD and $D_{2} O$

Molecular structure of GLYD is shown in Fig.A, and the amide units are denoted as "a" and "b". In Fig.C, blue: water hydrogen; red: water oxygen. color online

重水溶液中具有良好的溶解能力。

\section{2 酰胺-I带光谱与结构相关性}

我们选取了在 $10 、 20$ 和 $30 \mathrm{ps}$ 时刻, GLYD 与 周围 $\mathrm{D}_{2} \mathrm{O}$ (水分子数 $\left.n=5\right)$ 所形成的聚集体的瞬态结
构, 在密度泛函理论水平上计算得到了 $3 N-6$ 个 振动模式的振动频率。其中, 处于 1660-1760 $\mathrm{cm}^{-1}$ 的酰胺-I带的振动吸收峰与分子结构具有显著的相 关性(图 2)。由于酰胺单元中两个 $\mathrm{C}=\mathrm{O}$ 基团倾向于

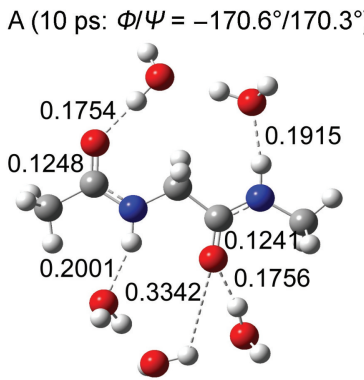

B (20 ps: $\left.\Phi / \Psi=65.3^{\circ} /-145.5^{\circ}\right)$
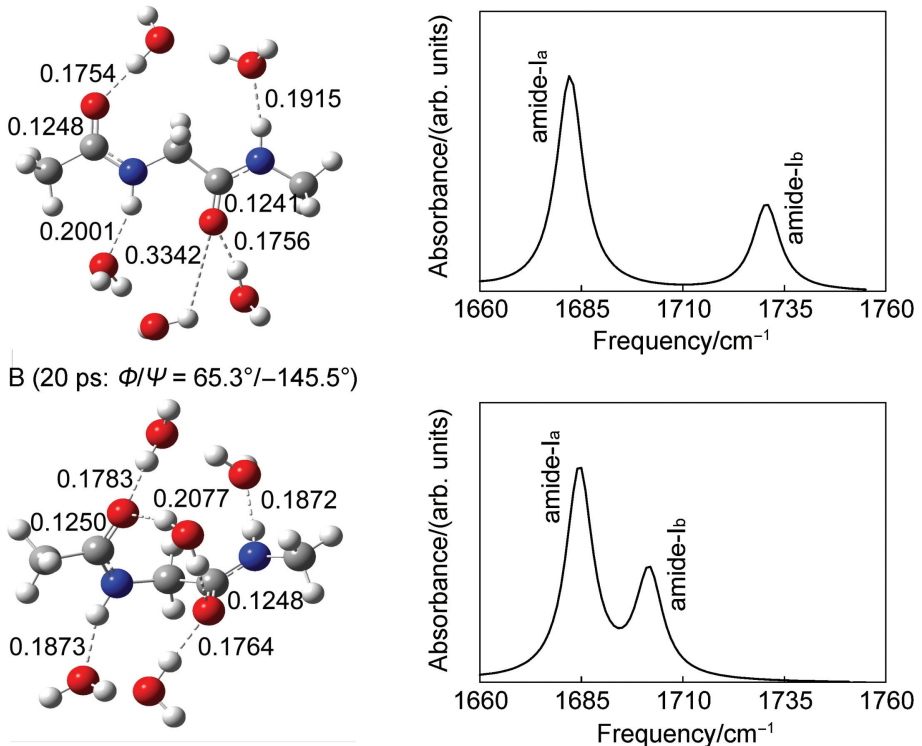

C $\left(30\right.$ ps: $\left.\Phi / \Psi=-86.2^{\circ} /-137.7^{\circ}\right)$
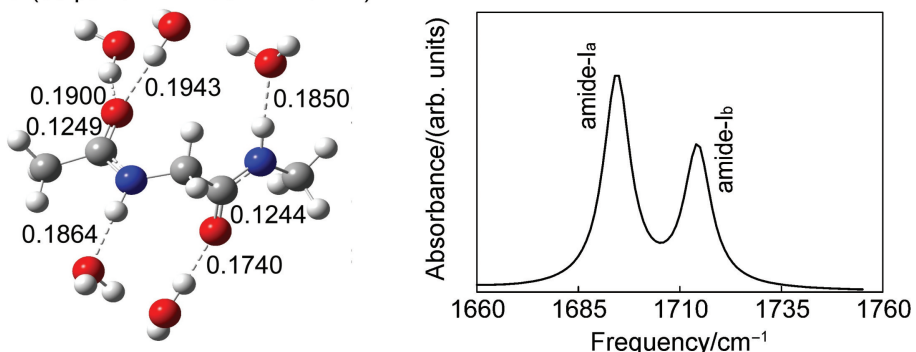

图 2 甘氨酸二肽与周围重水分子的瞬态结构及相应的酰胺-I带的计算光谱

Fig.2 Instantaneous structures of GLYD- $\mathrm{D}_{2} \mathrm{O}$ clusters and corresponding calculated amide-I spectra bond length in $\mathrm{nm}$ 
和重水形成较强的氢键作用, $\mathrm{C}=\mathrm{O}$ 双键的键长依 据氢键强弱的不同发生一定程度的拉伸, 并在酰 胺-I带的光谱区域产生相应的频率红移。

在动力学轨迹第 $10 \mathrm{ps}$ 的瞬态时刻(图 2A), 乙 酰端 $(\mathrm{C}=\mathrm{O}, 0.1248 \mathrm{~nm})$ a 的键长大于氨基端 $(\mathrm{C}=$ $\mathrm{O}, 0.1241 \mathrm{~nm})_{\mathrm{b}}$, 对应的酰胺 $-\mathrm{I}_{\mathrm{a}}$ 带的振动频率相比 酰胺- $\mathrm{I}_{\mathrm{b}}$ 带红移了 $48.4 \mathrm{~cm}^{-1}$; 在 $20 \mathrm{ps}$ 的瞬态时刻(图 2B), $(\mathrm{C}=\mathrm{O}, 0.1248 \mathrm{~nm})_{\mathrm{b}}$ 由于和周边的水分子同 时形成两个较强的氢键, 酰胺 $-\mathrm{I}_{\mathrm{b}}$ 带振动频率红移 至 $1701.7 \mathrm{~cm}^{-1}$, 与酰胺 $-\mathrm{I}_{\mathrm{a}}$ 带的频率差缩小至 17.2 $\mathrm{cm}^{-1}$; 在 $30 \mathrm{ps}$ 的瞬态时刻(图 2C), 溶质-溶剂间 氢键作用较弱, 两个 $\mathrm{C}=\mathrm{O}$ 双键键长较 $20 \mathrm{ps}$ 时有 所缩短, 酰胺-I带频率整体蓝移。随着时间的迁 移, GLYD及周围重水处于不停的热运动之中, 溶 质一溶剂相互作用对 GLYD 二级结构的影响在酰 胺-I带中表现出来, 使得光谱表象成为有力的结构 探测信号。

对等间隔选取的 GLYD- $n \mathrm{D}_{2} \mathrm{O}(n=1-5)$ 聚集体 开展量子化学计算得到优化后的结构及对应的酰 胺-I 带振动频率, 其相关性如图 3 所示。在 $140 \mathrm{ps}$ 瞬态时刻, 随着 GLYD 周围重水分子个数的增加, $\mathrm{C}=\mathrm{O}$ 键长由于氢键的作用而拉伸变长, 导致相应 的酰胺-I 带的振动频率发生的红移程度加剧(图 $3 \mathrm{C}), \mathrm{C}=\mathrm{O}$ 可能同时和两个重水分子分别形成分 子间氢键。对 120 个聚集体的酰胺-I带进行统计分 析, 发现酰胺- $\mathrm{I}_{\mathrm{a}}$ 带频率均值分布从 $1717.9 \mathrm{~cm}^{-1}$ (GLYD-1 $\mathrm{D}_{2} \mathrm{O}$ ) 至 $1703.5 \mathrm{~cm}^{-1}\left(\mathrm{GLYD}-5 \mathrm{D}_{2} \mathrm{O}\right)$, 酰胺- $\mathrm{I}_{\mathrm{b}}$ 带频率均值分布从 $1741.3 \mathrm{~cm}^{-1}\left(1 \mathrm{D}_{2} \mathrm{O}\right)$ 至 1695.9 $\mathrm{cm}^{-1}\left(5 \mathrm{D}_{2} \mathrm{O}\right)$ 。在 GLYD- $5 \mathrm{D}_{2} \mathrm{O}$ 聚集体外层添加了 PCM 模型补偿溶液体相作用后, 酰胺-I带振动频
率仍有一定程度的红移, 频率均值分别红移至 $1664.5 \mathrm{~cm}^{-1}$ (酰胺- $\mathrm{I}_{\mathrm{a}}$ 带) 和 $1668.5 \mathrm{~cm}^{-1}$ (酰胺 $-\mathrm{I}_{\mathrm{b}}$ 带)。 溶剂产生的酰胺-I带频率红移主要来自第一水合层 内重水所产生的氢键作用, 但是来自体相的作用 不可忽视。

酰胺-I带光谱的频移除了受到溶质－溶剂间氢 键作用的影响, GLYD本身二级结构的构象态变化 也会导致光谱吸收带的迁移。通过对气相中遍及 整个拉式构象图的孤立的 GLYD构象异构体进行简 正模式分析, 并借助 PED 分析方法对酰胺-I 带进行 了系统的指认和归属, 得到了气相中 GLYD 分子两 个酰胺-I带的振动频率随分子二级结构变化的规律 (图 4)。

酰胺 $-\mathrm{I}_{a} /-\mathrm{I}_{b}$ 带频率在拉式图中沿反对角线呈对 称分布。其中, 酰胺 $-\mathrm{I}_{\mathrm{a}}$ 带的频率分布范围从 $1722.3 \mathrm{~cm}^{-1}\left(\Phi / \Psi=80^{\circ} /-70^{\circ}\right)$ 至 $1824.0 \mathrm{~cm}^{-1}(\Phi / \Psi=$ $\left.0^{\circ} / 180^{\circ}\right)$, 酰胺- $\mathrm{I}_{\mathrm{b}}$ 带的频率则分布在 $1720.4 \mathrm{~cm}^{-1}(\Phi /$ $\left.\Psi=-10^{\circ} / 10^{\circ}\right)$ 至 $1796.1 \mathrm{~cm}^{-1}\left(\Phi / \Psi=-50^{\circ} /-90^{\circ}\right)$ 。 酰胺 $-\mathrm{I}_{\mathrm{a}}$ 带的振动频率平均值 $\left(1760.6 \mathrm{~cm}^{-1}\right)$ 略高于酰 胺- $\mathrm{I}_{\mathrm{b}}$ 带 $\left(1758.0 \mathrm{~cm}^{-1}\right)$ 。简正模式分析结果揭示了 GLYD 各个构象异构体均有特异性的酰胺-I带指纹 吸收频率, 且简正模式包含了可能存在的振动耦 合、费米共振等影响光谱频移的因素。如此构建 的构象异构体的酰胺-I 带频率数据库能够有效地将 二级结构变化以光谱表象的形式进行表达。

在气相或是溶液中, 分子的热运动导致 GLYD 的结构涨落遵循一定的规律, 分子倾向于形成较 为稳定的构象。在气相中, 孤立的 GLYD 的两个酰 胺单元倾向于形成分子内氢键, 使得分子呈现 $\mathrm{C}_{5}$ 和 $\mathrm{C}_{7}$ 构象 ${ }^{39}$ 。而在水溶液中, 酰胺单元和水分子间
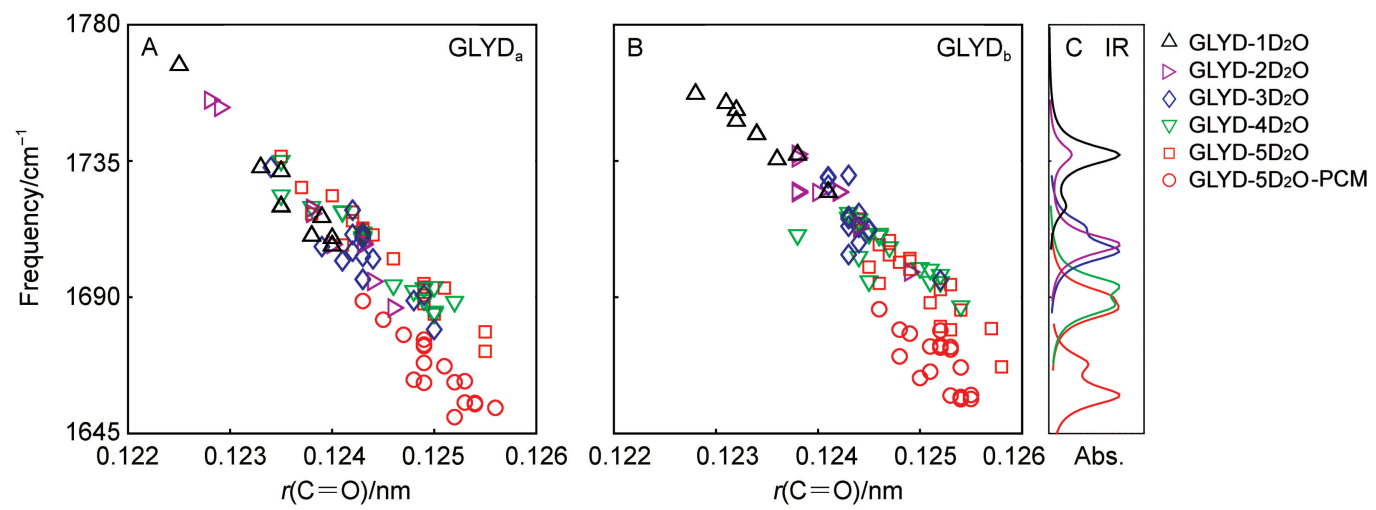

图 3 酰胺-I带频率与 $C=O$ 键长的相关性 $(A, B)$ 及第 $140 \mathrm{ps}$ 瞬态时刻 GLYD- $n \mathrm{D}_{2} \mathrm{O}$ 的酰胺-I带 IR 光谱 $(C)$

Fig.3 Correlation between the amide-I frequencies and the $C=O$ bond lengths $(A, B)$, and the calculated IR spectra of amide-I mode for selected GLYD- $n \mathrm{D}_{2} \mathrm{O}$ clusters at $140 \mathrm{ps}(\mathrm{C})$

Abs.: normalized absorbance. color online 


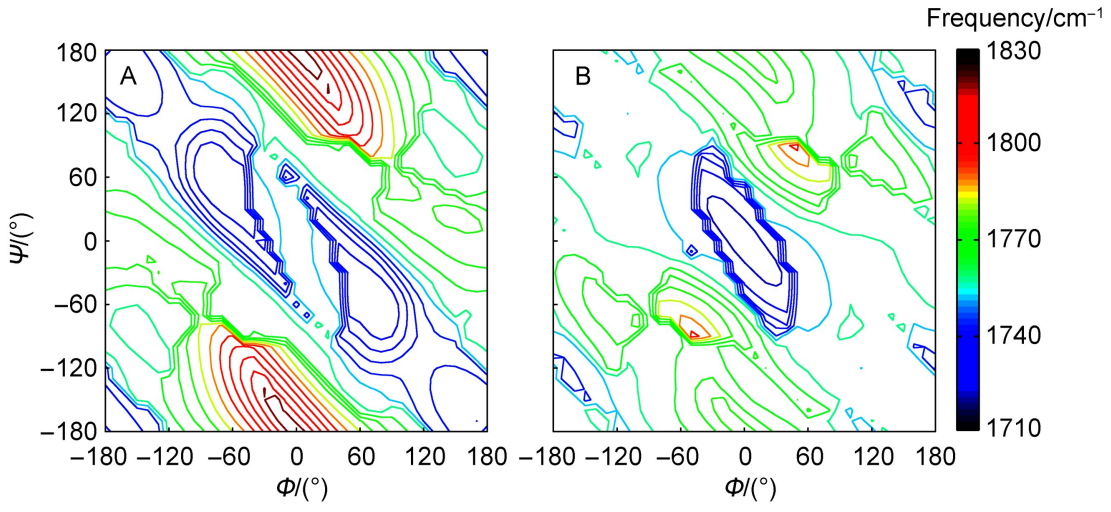

图 4 酰胺-I 带振动频率随 GLYD 骨架二面角 $(\Phi / \Psi)$ 变化分布

Fig.4 Amide-I frequency distributions due to the backbone dihedrals ( $\Phi / \Psi)$ of GLYD

(A) amide- $\mathrm{I}_{\mathrm{a}}$; (B) amide- $\mathrm{I}_{\mathrm{b}}$. color online

存在较强的氢键作用, 分子动力学模拟结果表明 GLYD 倾向于形成 $\mathrm{PP}_{\mathrm{II}}, \beta$-折叠, $\alpha$-螺旋等构象。 由于绝大多数生命活动都是在溶液环境下发生 的, 要考虑溶液相中溶剂作用对光谱的影响, 则 需要将极性溶剂环境下占主导地位的静电作用进 行量化。同时, 溶剂作用使得 GLYD 构象态发生改 变, 溶剂作用隐性地在结构中发生作用, 此时将 上述简正模式分析中构象态对光谱参数的影响引 入静电频率转换图中(公式(1), $\left.\omega_{(\Phi, 4)}\right)$, 使其具有二 级结构的敏感性, 结合溶剂中占主导地位的静电作 用, 与气/液相中酰胺-I带频移相关联, 从而计算 获取转换图参数。借助模型方法, 只需知道多肽所 呈现的折叠特性及溶剂分子在酰胺单元上所产生的 静电势场作用, 就能够有效实现多肽乃至蛋白质特 定位点酰胺-I带振动吸收峰的快速准确预测。

\section{3 酰胺-I带光谱模拟}

计算整个动力学轨迹中溶剂和 GLYD 骨架在酰 胺单元上所产生的静电势场作用, 结合气相中酰 胺-I带的二级结构依赖性, 将模型参数(表 1)应用 于整个分子动力学轨迹中, 得到优化后的 GLYD 中 酰胺-I 带的频率统计分布(图 5, DOS), 进一步通 过线型函数(方程(2))可以计算得到其红外吸收光谱 (图 5, IR)。

经过模型优化得到两个酰胺-I带的最可几频率 分别位于 $1631 \mathrm{~cm}^{-1}$ (酰胺- $\mathrm{I}_{\mathrm{a}}$ ) 和 $1646 \mathrm{~cm}^{-1}$ (酰胺$\mathrm{I}_{\mathrm{b}}$ ), 其统计分布均呈现高斯型分布, 拟合得到的半 高全宽 (FWHM) 分别为 $34.1 \mathrm{~cm}^{-1}$ (酰胺 $-\mathrm{I}_{\mathrm{a}}$ ) 和 34.0 $\mathrm{cm}^{-1}$ (酰胺- $\mathrm{I}_{\mathrm{b}}$ ), 且均位于实验光谱吸收带的范围 内。由于 GLYD 的 $\mathrm{C}_{\alpha}$ 上的 $\mathrm{R}$ 基仅有 $\mathrm{H}$ 原子, 分子 具有一定的对称性，因此水溶液中 GLYD 的 FTIR
光谱仅呈现出一个振动吸收峰(图 5) ${ }^{40}$ 。为了便于和 实验值比较, 将两个酰胺-I带的频率轨迹进行了叠 加之后进行统计分析(图 5, Sum-DOS), 其最可几 分布值 $\left(1639.0 \mathrm{~cm}^{-1}\right)$ 较好地重现了实验光谱的吸收 峰值 $\left(1640.3 \mathrm{~cm}^{-1}\right)$, 表明实验测试得到的 GLYD 光 谱虽然表现为单峰, 但实际上是由两个十分接近的 组分构成, 两个吸收峰对应的化学结构有所不同。

然而 DOS 并不是真实的红外光谱, 由于未考 虑到运动窄化作用 ${ }^{41,42}$, 酰胺-I带的谱带宽度(SumDOS， $\left.37.7 \mathrm{~cm}^{-1}\right)$ 大于其实验观测值 $\left(33.5 \mathrm{~cm}^{-1}\right)$ 。通 过线型函数对两个酰胺-I带分别进行了 IR 模拟, 所得到的局域模式(图 5)的吸收峰频率和统计分布 的最可几频率一致, 其 FWHM 相较对应的 DOS 有 一定程度的窄化, 分别为 $22.2 \mathrm{~cm}^{-1}$ (酰胺- $\mathrm{I}_{\mathrm{a}}$ ) 和 23.6 $\mathrm{cm}^{-1}$ (酰胺- $\left.\mathrm{I}_{\mathrm{b}}\right)$ 。同时, 考虑到两个酰胺-I 带之间的

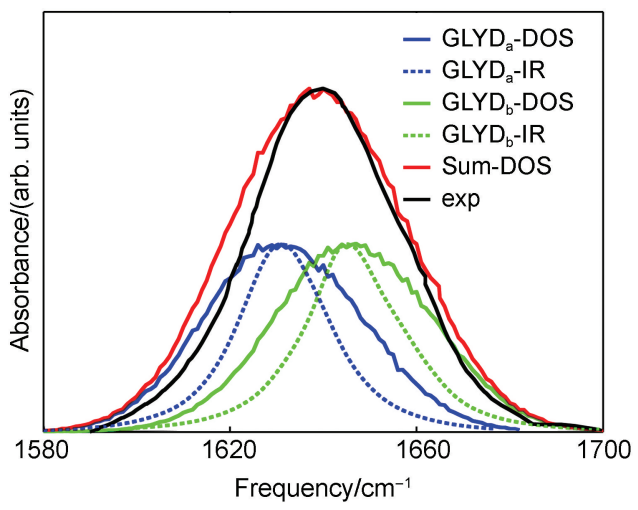

图 5 重水溶液中 GLYD 两个酰胺-I 带频率的统计分布及 模拟红外光谱

Fig.5 Static frequency distributions and the simulated IR spectra for the amide- $I_{a}$ and $-I_{b}$ modes of GLYD in $D_{2} O$ The experimental spectrum is taken from Cormanich et al. ${ }^{40}$. DOS: density of state; Sum-DOS: sum of density of state. color online 
振动耦合, 我们还采用此耦合方法进行了光谱的 模拟 ${ }^{43}$, 然而该方法并不适用于现有模型得到的频 率轨迹, 得到的红外光谱具有明显的双峰特性(数 据未给出)。此外, 在构建模型时已经引入了酰胺I 带简正模式的二级结构依赖性, 在一定程度上包 含了振动模式之间的耦合作用。模型的建立能够 有效实现光谱参数的快速预测, 借助模型参数获 得光谱数据的计算量远远小于量子化学计算, 同 时不受到样本大小、计算方法、基组水平的影 响, 并能够帮助人们解析实验光谱信号在真实世 界中所表达的物理化学意义, 进而依据光谱表 象, 有效描述溶液中多肽的结构特性及所处化学 微环境特征。

\section{4 结 论}

溶液相中蛋白质和多肽空间结构的解析一直 是一个研究热点。本文结合量子化学计算方法和 分子动力学模拟手段, 获取了飞秒至纳秒时间尺 度下甘氨酸二肽的结构动力学特性。通过空间分 布函数和径向分布函数, 对可能存在的溶质 - 溶剂 相互作用有了系统的认识。提取了分子动力学轨 迹中不同瞬态时刻分子聚集体开展量子化学计 算, 了解溶质一溶剂间相互作用对多肽二级结构的 影响及其相关的光谱表象, 进一步等间隔选取了 分子动力学轨迹中具有代表性结构的 GLYD- $n \mathrm{D}_{2} \mathrm{O}$ 聚集体, 探索溶剂作用对酰胺-I带光谱频移的影 响。考察气相中孤立 GLYD 各个构象异构体的酰 胺-I带的简正振动频率, 认识光谱表象的二级结构 依赖性及其潜在的振动耦合等作用。建立起溶剂 静电作用、酰胺-I带简正模式随构象态变化规律与 酰胺-I带在气/液相中光谱频移之间的相关性, 构 造具有二级结构敏感性的静电频率转换模型, 实 现溶液相中对多肽二级结构敏感的酰胺-I带光谱参 数的快速准确预测。

致 谢: 感谢中国科学院超算中心网格计算提供的高性能 计算服务。

\section{References}

(1) Carrell, R. W.; Lomas, D. A. Lancet 1997, 350, 134. doi: 10.1016/S0140-6736(97)02073-4

(2) Savelieff, M. G.; DeToma, A. S.; Derrick, J. S.; Lim, M. H. Accoutns Chem. Res. 2014, 47, 2475. doi: 10.1021/ar500152x

(3) Dill, K. A.; MacCallum, J. L. Science 2012, 338, 1042. doi: 10.1126/science. 1219021

(4) DeToma, A. S.; Salamekh, S.; Ramamoorthy, A.; Lim, M. H. Chem. Soc. Rev. 2012, 41, 608. doi: 10.1039/C1CS15112F

(5) Krimm, S.; Bandekar, J. Adv. Protein Chem. 1986, 38, 181. doi: 10.1016/S0065-3233(08)60528-8

(6) Barber-Armstrong, W.; Donaldson, T.; Wijesooriya, H.; Silva, R. A. G. D.; Decatur, S. M. J. Am. Chem. Soc. 2004, 126, 2339. doi: $10.1021 / \mathrm{ja} 037863 \mathrm{n}$

(7) Huang, C. Y.; Getahun, Z.; Zhu, Y.; Klemke, J. W.; DeGrado, W. F.; Gai, F. Proc. Natl. Acad. Sci. U. S. A. 2002, 99, 2788. doi: 10.1073/pnas.052700099

(8) Du, D.; Zhu, Y.; Huang, C. Y.; Gai, F. Proc. Natl. Acad. Sci. U. S. A. 2004, 101, 15915. doi: 10.1073/pnas.0405904101

(9) Malolepsza, E.; Straub, J. E. J. Phys. Chem. B 2014, 118, 7848. doi: 10.1021/jp412827s

(10) Woys, A. M.; Almeida, A. M.; Wang, L.; Chiu, C. C.; McGovern, M.; de Pablo, J. J.; Skinner, J. L.; Gellman, S. H.; Zanni, M. T. J. Am. Chem. Soc. 2012, 134, 19118. doi: 10.1021/ja3074962

(11) Kim, Y. S.; Wang, J.; Hochstrasser, R. M. J. Phys. Chem. B 2005, 109, 7511. doi: 10.1021/jp044989d

(12) Moran, S. D.; Zanni, M. T. J. Phys. Chem. Lett. 2014, 5, 1984. doi: $10.1021 / \mathrm{jz} 500794 \mathrm{~d}$

(13) Jones, K. C.; Peng, C. S.; Tokmakoff, A. Proc. Natl. Acad. Sci. U. S. A. 2013, 110, 2828. doi: 10.1073/pnas. 1211968110

(14) Kim, H.; Cho, M. Chem. Rev. 2013, 113, 5817. doi: 10.1021/ cr3005185

(15) Tucker, M. J.; Abdo, M.; Courter, J. R.; Chen, J.; Brown, S. P.; Smith, A. B.; Hochstrasser, R. M. Proc. Natl. Acad. Sci. U. S. A. 2013, 110, 17314. doi: 10.1073/pnas. 1311876110

(16) Kim, Y. S.; Hochstrasser, R. M. J. Phys. Chem. B 2009, 113, 8231. doi: $10.1021 / \mathrm{jp} 8113978$

(17) Wang, J. P. Chin. Sci. Bull. 2007, 52, 1221. [王建平. 科学通 报, 2007, 52, 1221.]

(18) Zheng, J. R. Physics 2010, 39, 162. [郑俊荣. 物理, 2010, 39, 162.]

(19) Carr, J. K.; Zabuga, A. V.; Roy, S.; Rizzo, T. R.; Skinner, J. L. J. Chem. Phys. 2014, 140, 224111. doi: 10.1063/1.4882059

(20) Jansen, T. L. C. J. Phys. Chem. B 2014, 118, 8162. doi: $10.1021 / \mathrm{jp} 5012445$

(21) Reppert, M.; Tokmakoff, A. J. Chem. Phys. 2013, 138, 134116/ 1. doi: $10.1063 / 1.4798938$

(22) Lin, Y. S.; Shorb, J. M.; Mukherjee, P.; Zanni, M. T.; Skinner, J. L. J. Phys. Chem. B 2009, 113, 592. doi: 10.1021/jp807528q

(23) Wang, L.; Middleton, C. T.; Zanni, M. T.; Skinner, J. L. J. Phys. Chem. B 2011, 115, 3713. doi: 10.1021/jp200745r

(24) Dijkstra, A. G.; Jansen, T. L. C.; Knoester, J. J. Phys. Chem. B 2011, 115, 5392. doi: 10.1021/jp109431a

(25) Lee, H.; Choi, J. H.; Cho, M. J. Chem. Phys. 2012, 137, 114307. doi: 10.1063/1.4751477

(26) Reppert, M.; Tokmakoff, A. J. Chem. Phys. 2015, 143, 061102. 
doi: $10.1063 / 1.4928637$

(27) Cai, K.; Su, T.; Lin, S.; Zheng, R. Spectrochim. Acta A 2014, 117, 548. doi: 10.1016/j.saa.2013.08.058

(28) Shi, J. P.; Zhao, J.; Yang, F.; Wang, J. P. Acta Phys. -Chim. Sin. 2013, 29, 695. [石纪培, 赵 娟, 杨 帆, 王建平. 物理化学学 报, 2013, 29, 695.] doi: 10.3866/PKU.WHXB201302213

(29) Cai, K.; Du, F.; Zheng, X.; Liu, J.; Zheng, R.; Zhao, J.; Wang, J. J. Phys. Chem. B 2016, 120, 1069. doi: 10.1021/acs. jpcb.5b11643

(30) Jansen, T. L. C.; Knoester, J. J. Phys. Chem. B 2006, 110, 22910. doi: 10.1021/jp064795t

(31) Phillips, J. C.; Braun, R.; Wang, W.; Gumbart, J.; Tajkhorshid, E.; Villa, E.; Chipot, C.; Skeel, R. D.; Kale, L.; Klaus, S. J. Comput. Chem. 2005, 26, 1781. doi: 10.1002/jcc.20289

(32) MacKerell, A. D., Jr.; Bashford, D.; Bellott, M.; Dunbrack, R. L., Jr.; Evanseck, J. D.; Field, M. J.; Fischer, S.; Gao, J.; Guo, H.; Ha, S.; Joseph-McCarthy, D.; Kuchnir, L.; Kuczera, K.; Lau, F. T. K.; Mattos, C.; Michnick, S.; Ngo, T.; Nguyen, D. T.; Prodhom, B.; Reiher, W. E., III; Roux, B.; Schlenkrich, M.; Smith, J. C.; Stote, R.; Straub, J.; Watanabe, M.; WiorkiewiczKuczera, J.; Yin, D.; Karplus, M. J. Phys. Chem. B 1998, 102, 3586. doi: 10.1021/jp973084f

(33) Jorgensen, W. L.; Chandrasekhar, J.; Madura, J. D.; Impey, R.
W.; Klein, M. L. J. Chem. Phys. 1983, 79, 926. doi: 10.1063/ 1.445869

(34) Jamróz, M. H. Vibrational Energy Distribution Analysis VEDA 4; Warsaw: Poland, 2004-2010.

(35) Frisch, M. J.; Trucks, G. W.; Schlegel, H. B.; et al. Gaussian 09, Revision A.01; Gaussian Inc.: Wallingford, CT, 2009.

(36) Schmidt, J. R.; Corcelli, S. A.; Skinner, J. L. J. Chem. Phys. 2004, 121, 8887. doi: 10.1063/1.1791632

(37) Papamokos, G. V.; Demetropoulos, I. N. J. Phys. Chem. A 2004, 108, 7291. doi: 10.1021/jp049563d

(38) Kim, Y. S.; Hochstrasser, R. M. J. Phys. Chem. B 2005, 109, 6884. doi: $10.1021 / \mathrm{jp} 0449511$

(39) Pohl, G.; Perczel, A.; Vass, E.; Magyarfalvi, G.; Tarczay, G. Phys. Chem. Chem. Phys. 2007, 9, 4698. doi: 10.1039/ b705098d

(40) Cormanich, R. A.; Rittner, R.; Buhl, M. RSC Adv. 2015, 5, 13052. doi: $10.1039 /$ C4RA16472E

(41) Saven, J. G.; Skinner, J. L. J. Chem. Phys. 1993, 99, 4391. doi: 10.1063/1.466092

(42) Kubo, R. Advances in Chemical Physcis; John Wiley \& Sons, Inc.: New York, 2007; p 101.

(43) Han, C.; Wang, J. ChemPhysChem 2012, 13, 1522. doi: 10.1002/cphc.v13.6 